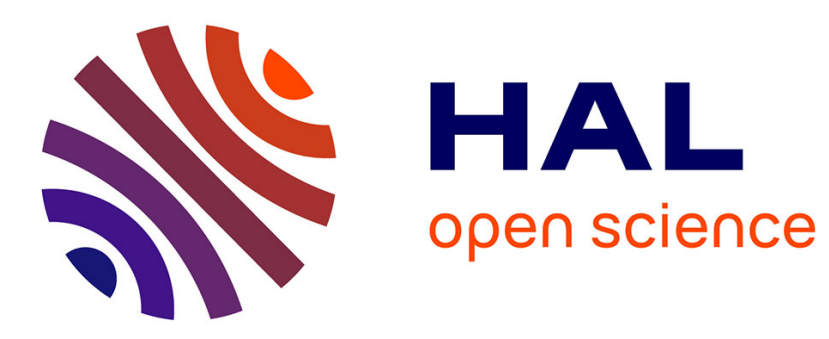

\title{
Simulated Raman spectra of bulk and low-dimensional phosphorus allotropes
}

\author{
A. Impellizzeri, A. Vorfolomeeva, N. Surovtsev, A. Okotrub, C. Ewels, D. \\ Rybkovskiy
}

\section{- To cite this version:}

A. Impellizzeri, A. Vorfolomeeva, N. Surovtsev, A. Okotrub, C. Ewels, et al.. Simulated Raman spectra of bulk and low-dimensional phosphorus allotropes. Physical Chemistry Chemical Physics, 2021, 23 (31), pp.16611-16622. 10.1039/d1cp02636d . hal-03356019

\section{HAL Id: hal-03356019 \\ https://hal.science/hal-03356019}

Submitted on 8 Oct 2021

HAL is a multi-disciplinary open access archive for the deposit and dissemination of scientific research documents, whether they are published or not. The documents may come from teaching and research institutions in France or abroad, or from public or private research centers.
L'archive ouverte pluridisciplinaire HAL, est destinée au dépôt et à la diffusion de documents scientifiques de niveau recherche, publiés ou non, émanant des établissements d'enseignement et de recherche français ou étrangers, des laboratoires publics ou privés. 


\title{
Simulated Raman spectra of Bulk and Low-Dimensional Phosphorus Allotropes
}

\author{
A. Impellizzeri ${ }^{1}$, A. A. Vorfolomeeva ${ }^{2}$, N. V. Surovtsev ${ }^{3}$, \\ A. V. Okotrub ${ }^{2}$, C. P. Ewels ${ }^{1 *}$, D. Rybovskiiy ${ }^{4 *}$ \\ ${ }^{1}$ Université de Nantes, CNRS, Institut des Matériaux Jean Rouxel, IMN, F-44000 Nantes, France \\ ${ }^{2}$ Nikolaev Institute of Inorganic Chemistry, SB RAS, 3 Acad. Lavrentiv Ave., \\ 630090 Novosibirsk, Russia \\ ${ }^{3}$ Institute of Automation and Electrometry SB RAS, 1 Acad. Koptyg Pr., \\ 630090 Novosibirsk, Russia
}
${ }^{4}$ Skolkovo Institute of Science and Technology, Bolshoy Boulevard 30, bld. 1, 121205 Moscow, Russia
*Corresponding authors: chris.ewels@cnrs-imn.fr rybkovskiyd@gmail.com

\begin{abstract}
We present a comprehensive theoretical and experimental Raman spectroscopic comparative study of bulk Phosphorus allotropes (white, black, Hittorf's, Fibrous) and their monolayer equivalents, demonstrating that the application of the Placzek approximation to density functional theory calculated frequencies allows reliable and accurate reproduction of the bulk spectra at a relatively low computational cost. As well as accurate frequencies, peak intensities are also reproduced with reasonable accuracy. Having established the viability of the method we apply it to other less well characterised phosphorus forms such as isolated $\mathrm{P}_{4}$ cages and the planar blue-phosphorus phase. There are several speculative structural models in the literature for amorphous red phosphorus, and we predict Raman spectra for several of these. Via comparison with experiment this allows us to eliminate many of them such as the $\mathrm{P}_{2} \mathrm{P}_{2}$-zigzag chain and connected $\mathrm{P}_{4}$ models. The combination of Density functional theory (DFT) modelling, Placzek approximation for intensities with experimental Raman spectroscopy is demonstrated as a powerful combination for accurate characterisation of phosphorus species.
\end{abstract}

Keywords: phosphorus; allotrope; Raman scattering; density functional theory; Placzek; vibrational modes 


\section{Introduction}

Amongst 2D materials, phosphorus $(\mathrm{P})$ is receiving increased recent interest. This is mainly due to the plethora of allotropic configurations existing in nature, such as black, white, red, violet, and their structural variants ${ }^{1}$. All of these structures are characterized by different properties, which make phosphorus suitable for a wide range of applications. For example, black phosphorus can been used in transistors, optoelectronics, sensors, and batteries, due to its high carrier mobility combined with thickness dependent band gap ${ }^{2}$, ranging from 0.31-0.36 eV to $1.45 \mathrm{eV}$ for 3D bulk and its 2D exfoliated single-layer counterpart (known as phosphorene), respectively ${ }^{3}$.

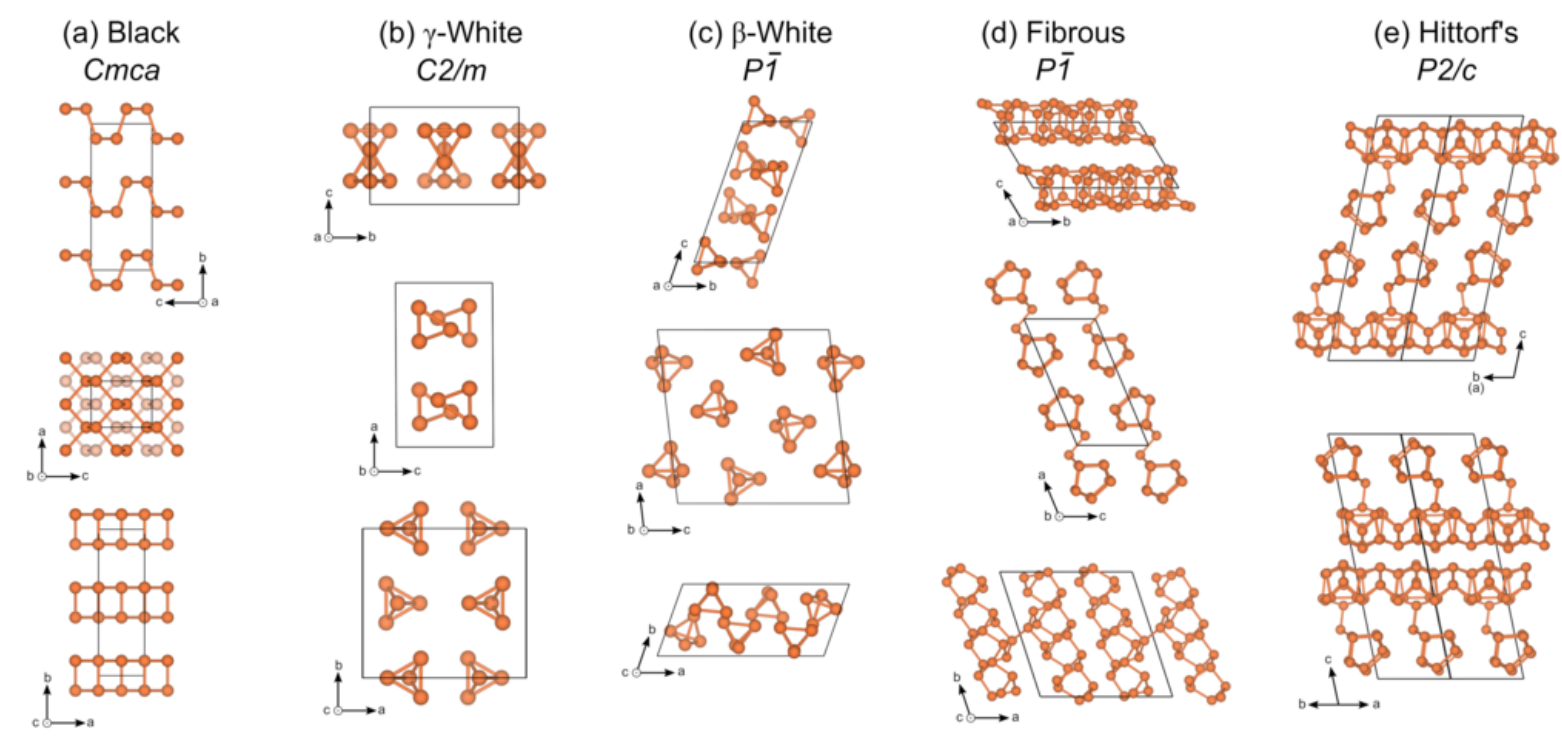

Figure 1: Crystal structures of bulk phosphorus allotropes (a) black, (b) $\gamma$-white, (c) $\beta$-white, (d) fibrous (red), (e) Hittorf's with different orientations with associated space group. Black lines represent the conventional unit cell for each configuration.

Table 1. Bulk phosphorus allotropes, with associated space group and $Z$ the number of atoms in the primitive unit cells. When two space groups are given, the second refers to the monolayer. The Materials Project Id refers to the structure pages at materialsproject.org

\begin{tabular}{|c|c|c|c|c|}
\hline P allotrope & $\begin{array}{c}\text { Space Group } \\
\text { Bulk: } \\
\text { Monolayer }\end{array}$ & $\begin{array}{c}\text { Materials } \\
\text { Project Id }\end{array}$ & Z & Other names \\
\hline Blue (A7) & $R \overline{3} m: P \overline{3} m 1$ & mp-130 & 2 & \\
\hline
\end{tabular}




\begin{tabular}{|c|c|c|c|c|}
\hline Black (A17) & Cmce: Pmna & $\begin{array}{c}\mathrm{mp}-157: \\
\mathrm{mp}-101401\end{array}$ & 4 & \multirow{2}{*}{ Yellow } \\
\hline$\gamma$-white & $C 2 / m$ & $\mathrm{mp}-12883$ & 8 & \\
\hline$\beta$-white & $P \overline{1}$ & $\mathrm{mp}-118$ & 24 & Red-IV \\
\hline Fibrous red & $P \overline{1}$ & $\mathrm{mp}-1198724$ & 42 & Violet, Red-V \\
\hline Hittorf's & $P 2 / c$ & $\mathrm{mp}-568348$ & 84 & \\
\hline
\end{tabular}

Principle allotropes with well-characterized crystal structures are shown in Fig. 1 with details listed in Table 1. Black Phosphorus is a buckled layered phase with orthorhombic lattice symmetry ${ }^{4,5}$. Isolated layers have been produced and are commonly referred to as Phosphorene or black phosphorene. The crystalline form of white phosphorus (sometimes also referred to as Yellow or tetraphosphorus) is given by a combination of $\mathrm{P}_{4}$ molecules, whose arrangement depends on the temperature. At room temperature the arrangement is similar to the $\alpha-\mathrm{Mn}$ structure $^{6}$, while cooling below $195.2 \mathrm{~K}$, two new white phosphorus phases appear, $\beta$ - and $\gamma$-, where $\mathrm{P}_{4}$ molecules form triclinic and monoclinic arrangements, respectively ${ }^{6}$. Red phosphorus is composed from linked pentagonal tubes of phosphorus ${ }^{7}$. In Hittorf's phosphorus these chains are arranged in cross-linked bilayers with the fibres in each layer orthogonal to the next in a monoclinic arrangement. These bilayers can in principle be separated, with isolated bilayers referred to variously as Hittorfene or Hittorf's phosphorene. The other red $\mathrm{P}$ phase, where the chains are cross-linked in pairs and arranged parallel to each other with triclinic arrangement, is referred to as Fibrous red or Red-IV phosphorus ${ }^{7}$. At high pressure it is suggested that black phosphorus transforms to a simpler A7 phase ${ }^{8}$. This layered phase is also constructed from hexagonal sheets similar to black phosphorus, but in this case the buckling has higher symmetry, with alternate sites lying above and below the sheet plane. Such buckled hexagonal layers have been referred to as blue phosphorene'. Although not yet isolated individually, there is evidence of the single-layer version grown on an $\mathrm{Au}(111)$ substrate using molecular beam epitaxy ${ }^{10}$.

Given the diverse bonding possibilities in elemental phosphorus, it has been the subject of extensive further structural searches amongst the theoretical community. Various hypothetical bulk and layered $\mathrm{P}$ allotropes have been theoretically predicted ${ }^{11-14}$, notably using machine learning-force fields ${ }^{15}$. Nanoscale 1D nanoribbons and nanotubes also appear regularly in the 
literature $^{16}$, and new nanoscale phases have been identified, for example when phosphorus is confined within the cavity of carbon nanotubes ${ }^{17-19}$.

The stability of different $\mathrm{P}$ forms is a question of ongoing controversy. Earlier combustion calorimetry studies indicated that black phosphorus is the most stable phase at low temperature and pressure ${ }^{4,5}$, however recent calculations using van der Waals (vdW) inclusive density functional theory (DFT) find red P more stable than black ${ }^{20}$. There is currently no conclusive evidence for the relative stability of these phases at room temperature and pressure $^{21}$.

Part of the experimental complexity when studying phosphorus is to discriminate easily between different phases, particularly when either mechanically confined, produced in nanoscale quantities, or under extreme conditions of temperature or pressure. In this context Raman spectroscopy plays a pivotal role, as a powerful, non-destructive, and sensitive tool able in principle to differentiate all of the bulk phases described above ${ }^{22}$. Although traditionally a bulk characterization technique, significant advances in both sample manipulation, mapping, super resolution and signal deconvolution ${ }^{23,24}$ mean that Raman spectroscopy can now also be increasingly applied to individual nano-objects. In order to make the link between experimental Raman spectra and underlying atomic structure, accurate spectral modelling is required.

Generally, a full quantum mechanical treatment based on perturbation theory is recommended for an accurate quantitative description of Raman spectra of both molecules and solids ${ }^{25,26}$. However, the computational cost of this approach is heavy, which in practice limits its use to systems with few atoms, and large systems such as the Hittorf's phosphorus (having 84 atoms) studied here are not properly accessible with current computational resources.

Recently, we have used semi-classical Placzek approximation within DFT accuracy to describe Raman spectra of large-size carbon systems, showing excellent agreement with experiment ${ }^{27}$. The underlying idea of this approximation is that the Raman intensity is directly proportional to the derivatives of the dielectric tensors with respect to the vibrational coordinates $^{28}$. In this approach, we consider only first-order Raman processes in which a single phonon is involved.

In the current paper, we present calculated Raman spectra of key bulk phosphorus species using the Placzek approximation. The calculations are based on DFT implementation of the 
first-order Raman scattering process employing vdW dispersion corrections and a relativistic Gaussian basis set. This approach successfully reproduces experimental spectra for a variety of phases, giving confidence in its use as a predictive tool. We include calculated spectra for several as-yet unmeasured or speculated phosphorus phases. This study constitutes a reliable reference for experimentalists and theoreticians working in the field of characterization of phosphorus materials.

\section{Computational and Experimental Methods}

Density Functional Theory. All first-principle calculations are performed with the ab initio

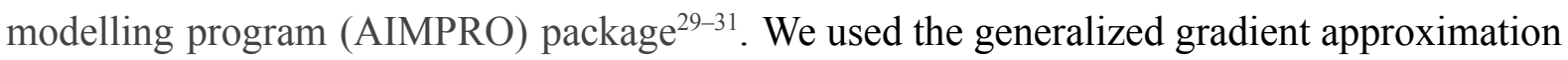
(GGA) with Perdew-Burke-Ernzerhof (PBE) formulation ${ }^{32}$. Van der Waals forces are included with D2 and D3 (zero damping) schemes developed by Grimme and co-workers ${ }^{33,34}$. The action of core electrons is modeled using the Hartwingsen-Goedecker-Hütter pseudopotentials $^{35}$. The electron wave functions have been expanded on a basis set of relativistic Gaussian orbitals ${ }^{36}$. The basis set used for phosphorus consists of a large number of $40 d$-type functions in order to provide results with maximum accuracy. A system-dependent plane-wave energy cutoff of $300 \mathrm{Ha}$ (Ha: Hartree energy) is taken, and a Fermi smearing of electronic occupations with an effective temperature of $0.02 \mathrm{eV}$ is set during the geometry optimization procedure.

We fully optimized atomic positions (including lattice vectors and angles) of each phosphorus structure by AIMPRO until the maximum atomic position change in a given iteration dropped below $10^{-6} \mathrm{a}_{0}$ ( $\mathrm{a}_{0}$ : Bohr radius) and evaluated the final total energy with tolerance set to $10^{-7}$ $\mathrm{Ha}$ in order to gauge the performance of $\mathrm{vdW}$ dispersion corrections mentioned above. Brillouin zone sampling uses a Monkhorst-Pack (MP) scheme with scalable $k$-points depending on the size of periodic lattice vectors and adopting periodic boundary conditions. Atomic structures are visualized using OVITO $^{37}$.

Raman Modelling. Once performed structural relaxation, phonon frequencies and eigenvectors at the $\Gamma$ point of phosphorus phases are calculated using AIMPRO code. The dynamical matrix is constructed on the basis of numerical second derivatives of the total energy with respect to the positions of atoms $i$ and $j$, which are obtained by a finite difference scheme from the calculated forces, with the finite atomic displacements of $0.106 \AA$. After the diagonalization of the dynamical matrix, a data set of atomic coordinates displaced along 
each of the mass-weighted phonon eigenvectors is generated, considering both positive and negative displacements. For every displaced configuration, we have evaluated both real and imaginary part of the frequency-dependent dielectric tensor, $\varepsilon_{\alpha \beta}$, within the sum over states approach from the electronic band structure of the phosphorus systems at energies $\mathrm{E}_{\mathrm{exc}}$, close to the experimentally used excitation energies by using the AIMPRO software. For each structure, a scissor operator $S$ is applied to correct the band gap underestimation due to the semi-local DFT exchange-correlation functional. While black $\mathrm{P}$ is investigated with $S=0.6$ $\mathrm{eV}$, the computations for white and red species are made with a $S$ operator set to $0.7 \mathrm{eV}$. Both phonon and dielectric tensor calculations require a high level of accuracy, and for this reason we increased the convergence tolerance for self-consistency to $10^{-10} \mathrm{Ha}$.

The Raman tensor matrix elements $R_{\alpha \beta}\left(j, E_{e x c}\right)$ for phonon mode $j$ at excitation energy $E_{e x c}$ are then obtained from the derivatives of the dielectric tensor with respect to the mass-weighted eigenvector $Q_{j}$ :

$$
R_{\alpha \beta}\left(j, E_{e x c}\right) \propto \frac{\partial \varepsilon_{\alpha \beta}\left(E_{e x c}\right)}{\partial Q_{j}}
$$

The use of the energy-dependent dielectric tensor allows to cover resonance behavior of the Raman response.

After obtaining the dielectric function derivatives, the intensities are calculated averaging over all polarization directions of the incident and scattered $\operatorname{light}^{28}$ :

$$
I \propto \frac{\left(n_{j}+1\right)}{\omega_{j}}\left(45 a^{2}+7 \gamma^{2}+5 \delta^{2}\right)
$$

where: $n_{j}=\left(e^{\hbar \omega_{j} / k_{B} T}-1\right)^{-1}$ is the Boltzmann distribution function calculated at $\mathrm{T}=300 \mathrm{~K}$, $\omega_{j}$ is the vibrational frequency of mode $j, a$ is the mean polarizability, $\gamma$ represents the anisotropy, while $\delta$ indicates the asymmetric anisotropy. These three parameters are denoted as Raman invariants and they depend on the polarizations of incident and scattered light.

Once determined frequencies and Raman intensities, the final theoretical form of Raman spectra is obtained using a Lorentzian broadening set to $4.0 \mathrm{~cm}^{-1}$. 
Instrumental Methods. Powder samples were illuminated with $70 \mathrm{~mW}$ power of a $532 \mathrm{~nm}$ solid state laser. Illumination area was a stripe of $100 \times 1000 \mathrm{~m} \mu$ formed with spherical and cylindrical lenses. By this way the local laser intensity was significantly reduced in the comparison with the traditional focusing into a small area. Scattered light from the illuminated area was collected onto an entrance slit of a triple-grating Tri-Vista 777 spectrometer. In the case of the powder material inside an ampoule, the laser irradiation of $100 \mathrm{~mW}$ power was focused on the material by a lens with a focus of $60 \mathrm{~mm}$. Emission spectrum of a neon-discharge lamp was used for the wavelength calibration of the spectrometer. The spectral resolution was $4 \mathrm{~cm}^{-1}$.

\section{Results and Discussions}

\subsection{The Stability Range of Phosphorus Allotropes}

Before computing the Raman spectra, we first benchmark our calculated enthalpies of formation for the different allotropes against literature. Fig. 2 shows the relative energy stability of bulk P calculated using the semi-local GGA-PBE functional comparing the reliability of the two Grimme D2 and D3 schemes. Regarding white P, the $\gamma$-form is slightly more stable than its $\beta$-counterpart using vdW D2 corrections $(\sim 2.4 \mathrm{meV} / \mathrm{atom})$, or almost degenerate with D3 ( 1.0 meV/atom). This is consistent with the phase transformations observed experimentally by Okudera et $a l^{40}$. We have also double checked the energy stability of the $\beta$-phase by increasing the $k$-point meshes during structural optimization without any change. The relative stability between black and red phosphorus species is highly sensitive to the vdW scheme implemented in our GGA-PBE calculations. Black P is energetically favored with respect to the red P using the Grimme D2 correction. In contrast, black and red species are almost degenerate using the D3 version, with the Hittorf's red very slightly more stable ( 7.2 meV/atom). All of these results are in excellent agreement with those reported in literature $^{20}$, obtained through more sophisticated approaches including Tkatchenko and Scheffler (TS) scheme and even the random phase approximation (RPA) and summarized in Supplementary Information, Section 1, see Table S1. In summary we find that both D2 and D3 Grimme vdW schemes give acceptable levels of reproduction of structures and energetics, with very slight energetic preference for the D3 scheme. 


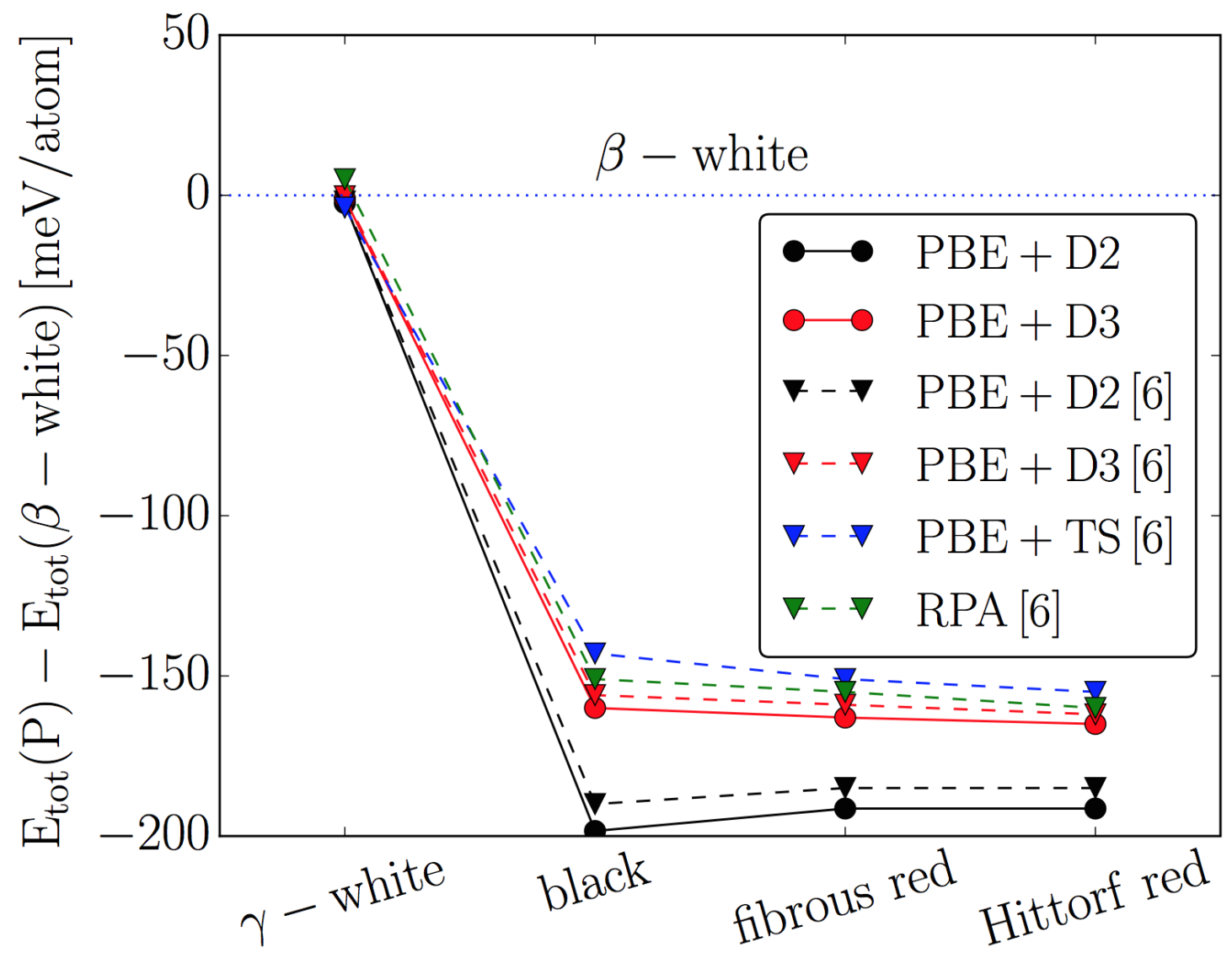

Figure 2: DFT-D calculated relative stability of $\gamma$-white, black, fibrous, and Hittorf's red allotropes of phosphorus with respect to the $\beta$-white phase. Dashed lines represent literature values obtained using the same and higher level methods, such as TS correction and RPA approach.

\subsection{Simulated Raman Spectra of Phosphorus Allotropes}

We next benchmark our theoretically determined Raman spectra against the key experimentally observed phosphorus phases, namely white, black and red phosphorus, as well as their associated single layer and molecular forms where appropriate. All calculated Raman spectra are for an excitation energy of $2.3 \mathrm{eV}(538 \mathrm{~nm})$, and experimental data for a laser excitation energy of $532 \mathrm{~nm}$ unless stated otherwise.

\subsubsection{White Phosphorus (molecular and bulk)}


All of the white phosphorus phases consist of $\mathrm{P}_{4}$ cages which then show different crystal packing (the room-temperature alpha-phase shows the additional complexity of rotational motional averaging of the $\mathrm{P}_{4}$ cages about their centre of mass). As such the key covalent bonding in the $\mathrm{P}_{4}$ tetrahedra of all these white phases is the same, with long range VdW type interaction determining the packing. Our calculated isolated $\mathrm{P}_{4}$ cage shows three distinct modes: the first at $371.5 \mathrm{~cm}^{-1}$ corresponds to the bending mode $\mathrm{E}$, while the higher frequency modes at 459.5 and $602.1 \mathrm{~cm}^{-1}$ are associated with symmetric stretching modes $F_{2}$ and $A_{1}$ (Supplementary Information, Section 3, Fig. S6).

Our experimental white alpha-P spectra are characterized by three very similar intense Raman active modes at 360.0, 457.0 and $597.0 \mathrm{~cm}^{-1}$. Fig. 3 shows the comparison of Raman intensities between data recorded in the experiment (black line) and DFT-PBE calculations for $\beta$-white phosphorus coupled with both Grimme D2 (red line) and D3 (blue line) corrections. Spectra measured at different time intervals after the start of the first measurements are reported in Supplementary Information, Section 3, see Fig. S5. The calculated spectrum is in good agreement with present and previous experimental data ${ }^{41,42}$. The only exception is a splitting in the calculated low-frequency E peak which is in contrast to the single peak observed experimentally. We attribute this splitting to the fact that the molecule packing within the room-temperature white-P allotrope is different from the calculated $\gamma$ - and $\beta$-phases, which appear at lower temperatures. Indeed, temperature-dependent Raman measurements performed on white-P revealed a splitting of the E-band between approximately 80 and $195 \mathrm{~K}^{17}$. The same authors reported a lower-frequency signal appearing synchronously with the splitting of the E mode at low temperatures with the peak positions at 48,70 , and $90 \mathrm{~cm}^{-1}$. Our calculations reproduce these features as a set of multiple low-frequency peaks that are merged when applying Lorenzian broadening. The most pronounced maxima of this signal are located at 15,32, 44, 84 and 90 $\mathrm{cm}^{-1}$. All these bands are produced by rigid-body oscillations and rotations of the $\mathrm{P}_{4}$ tetrahedra with respect to each other. Raman active modes for white $\mathrm{P}$ phases are summarized in Table 2.

Table 2. The three Raman active frequencies (in units of $\mathrm{cm}^{-1}$ ) in bulk white phosphorus recorded in the experiment and comparison with first-principle PBE $+D 2$ and D3 calculations under $2.3 \mathrm{eV}$ excitation energy: $O D$ isolated molecule, $3 D \alpha-, \beta$ - and $\gamma$-phase. Literature values from Ref ${ }^{41}$. 


\begin{tabular}{|c|c|c|c|c|}
\hline \multirow{3}{*}{ Configuration } & \multirow{2}{*}{ Source } & \multicolumn{3}{|c|}{ Frequency $\left(\mathrm{cm}^{-1}\right)$} \\
\cline { 3 - 5 } & & $\mathrm{E}$ & $\mathrm{F}_{2}$ & $\mathrm{~A}_{1}$ \\
\hline \multirow{3}{*}{$\alpha$} & Expt $^{41}$ & 360.6 & 458.0 & 598.8 \\
\cline { 2 - 5 } & Expt. $^{*} \beta$ & 360 & 457 & 597 \\
\hline \multirow{3}{*}{$\beta$} & Expt $^{41}$ & $355.8,363.5$ & 458.0 & 598.8 \\
\cline { 2 - 5 } & PBE+D2 & 359,368 & 456 & 594 \\
\cline { 2 - 5 } & PBE+D3 & 360,368 & 457 & 597 \\
\hline \multirow{3}{*}{$\gamma$} & Expt & 361.6 & 459.0 & 599.8 \\
\cline { 2 - 5 } & PBE+D2 & 351,364 & 454 & 592 \\
\cline { 2 - 5 } & PBE+D3 & 356,367 & 455 & 596 \\
\hline $\mathrm{P}_{4}$ Molecule & PBE+D2 & 372 & 460 & 602 \\
\cline { 2 - 5 } & PBE+D3 & 372 & 460 & 602 \\
\hline
\end{tabular}

a)

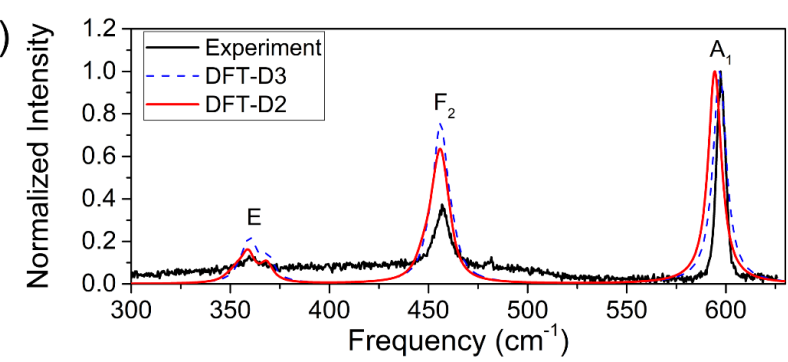

b)

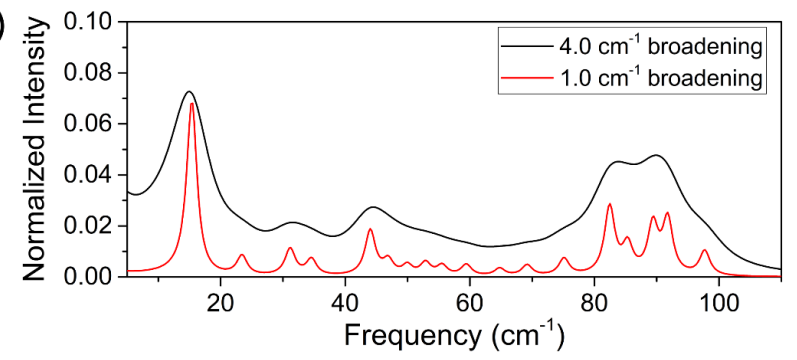

c)
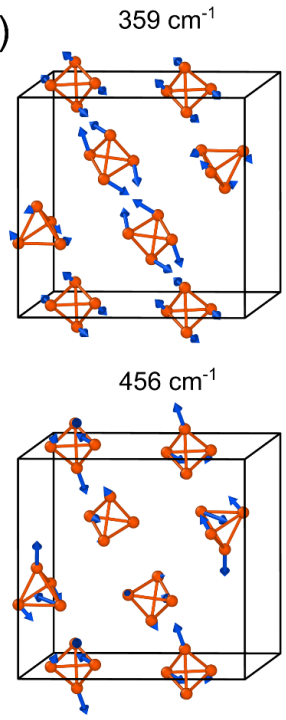
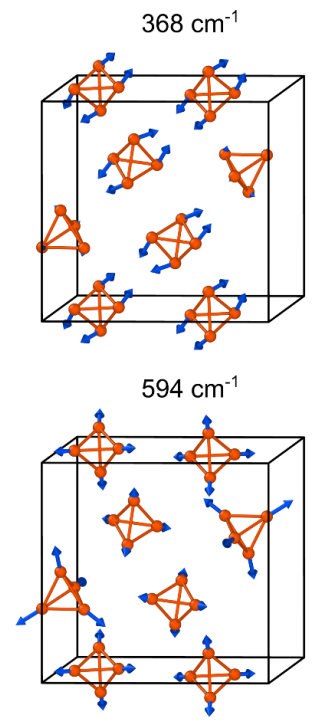
Figure 3: (a) DFT calculated Raman spectrum of bulk white phosphorus at 2.3eV excitation energy $(538 \mathrm{~nm})$ together with the recorded experimental spectrum (Black line). The spectra simulated through D2 and D3 corrections are indicated by red and blue lines, respectively. (b) The low-frequency part of the spectrum calculated within D2 with two different broadenings to show the fine-structure of each feature. (c) Calculated eigenvectors of key Raman active modes. Blue arrows indicate amplitude and direction of the displacements. Associated frequency is given in wavenumbers. Black lines indicate the symmetry of the simulated unit cell.

\subsubsection{Black Phosphorus (bulk and monolayer)}

Black Phosphorus is of great interest for semiconducting applications, because it exhibits a direct band gap tunable by changing the material thickness. The band gap estimated in previous experiments is around $0.33 \mathrm{eV}^{2,43-45}$. This value increases up to $\sim 2.0 \mathrm{eV}$ as the number of layers decreases for its exfoliated 1D counterpart, i.e. phosphorene ${ }^{3}$. In agreement with previous modelling, our dispersive DFT-vdW calculations show metallic character for bulk phosphorus (see Supplementary Information, Section 2, Fig. S1(a)) ${ }^{46}$, with band crossing from $\Gamma$ - to X-due to the use of semi-local exchange-correlation functionals. Our calculated single-layer phosphorene is semiconducting with a direct band gap of $0.89 \mathrm{eV}$ at the $\Gamma$-point (see Supplementary Information, Section 2, Fig. S1(b)), very close to literature values using both semi-local $\mathrm{PBE}^{47}$ and hybrid HSE06 ${ }^{3,47}$ functionals.

Our PBE+D2 simulated Raman spectra of bulk and single-layer black phosphorus are presented in Fig. 4(top). The bulk spectrum shows three pronounced peaks at $357.3 \mathrm{~cm}^{-1}$, $425.2 \mathrm{~cm}^{-1}$, and $453.5 \mathrm{~cm}^{-1}$, associated with $\mathrm{A}_{\mathrm{g}}{ }^{1}, \mathrm{~B}_{2 \mathrm{~g}}, \mathrm{~A}_{\mathrm{g}}{ }^{2}$ modes corresponding to the vibrations in the out-of-plane, zig-zag, and armchair directions, respectively (see Fig. 4(bottom)). These qualitatively match those recorded in our experiment and those reported in literature $3,45,48$.

Our calculated three peaks shift in frequency going from bulk to single-layer phosphorene, underlying a very small thickness-dependent dispersive behavior. Notably our experimental shift directions and magnitudes are very accurately reproduced by the calculations, with an upshift of the highest $\mathrm{A}_{\mathrm{g}}{ }^{2}$ mode, a smaller downshift of the lowest $\mathrm{A}_{\mathrm{g}}{ }^{1}$ mode, and a very slight downshift of the intermediate $\mathrm{B}_{2 \mathrm{~g}}$ mode (Table 3 ). We note there is some variability in these 
shifts in the literature ${ }^{3,49,50}$, probably due to different synthesis routes and supporting substrates for Phosphorene. The Raman active $\mathrm{A}_{\mathrm{g}}{ }^{1}$ and $\mathrm{A}_{\mathrm{g}}{ }^{2}$ peaks simulated via $\mathrm{PBE}+\mathrm{D} 2$ are close to those obtained in the experiment with respect to D3 correction. For $\mathrm{B}_{2 \mathrm{~g}}$ mode, a better quantitative agreement between simulation and experiment is obtained using the $\mathrm{PBE}+\mathrm{D} 3$ rather than D2 (see Fig. 4(top) and Table 3).
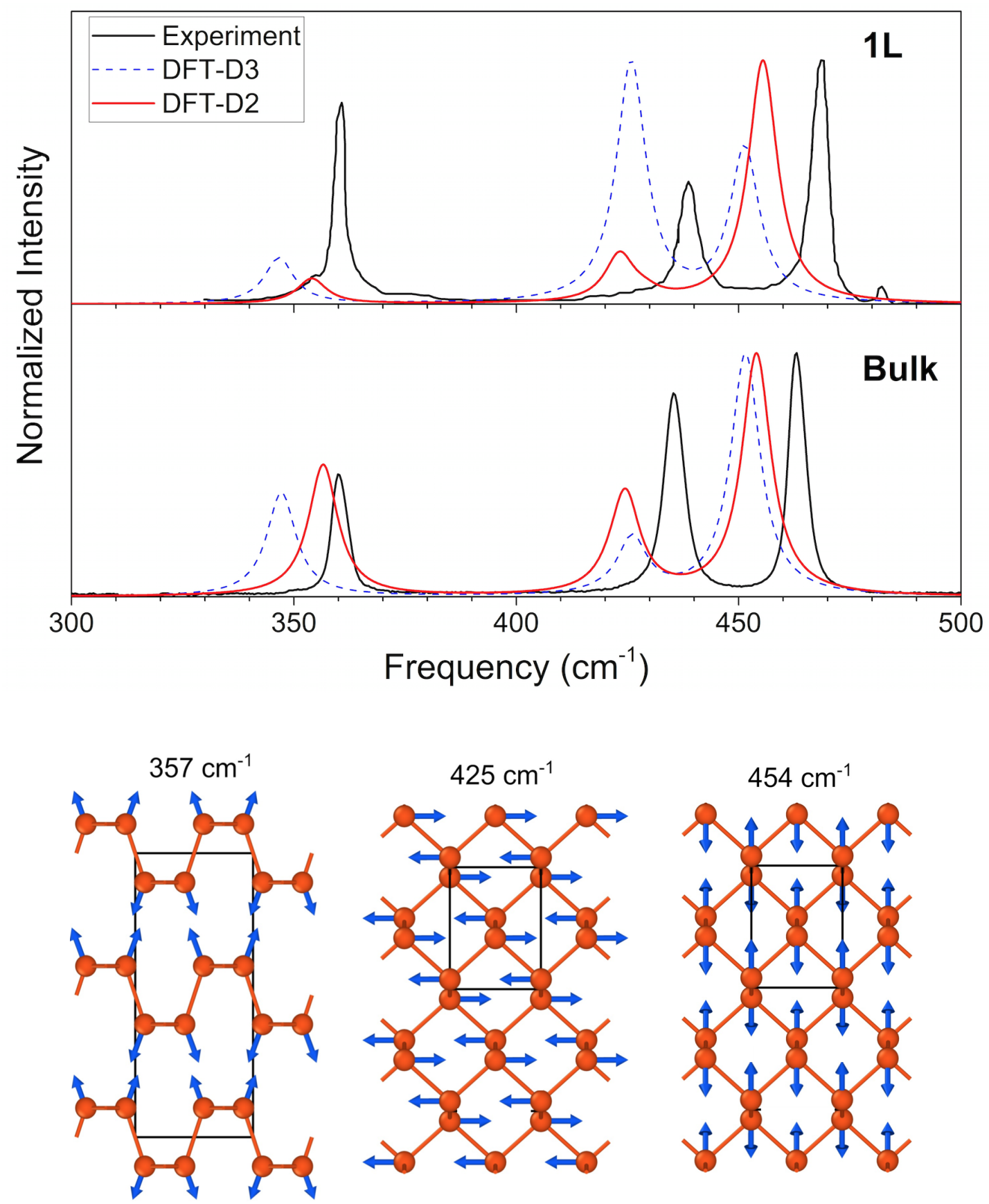

Figure 4: (Top) DFT calculated Raman spectrum of bulk (lower) and single layer (upper) black phosphorus at $2.3 \mathrm{eV}$ excitation energy $(538 \mathrm{~nm})$. The black line of the bulk case represents the spectrum recorded in our experiment. The black line of the single layer case is 
experimental data recorded by Liu et al. ${ }^{3}$ The spectra simulated through D2 and D3 corrections are indicated by red and blue lines, respectively. (Bottom) Calculated eigenvectors of key Raman active modes of bulk black phosphorus. Blue arrows indicate amplitude and direction of the displacements. Associated frequency is given in wavenumbers. Black lines indicate the symmetry of the simulated unit cell.

Table 3. Comparison of Raman active frequencies $\left(\mathrm{cm}^{-1}\right)$ in $3 D$ bulk black phosphorus and $2 D$ single-layer phosphorene, DFT PBE $+D 2$ and D3 values at $2.3 \mathrm{eV}$ excitation energy. Experimental values (532nm excitation) come from this work for bulk and compared with most recent measurements reported in literature ${ }^{48}$. For the single-layer case data are extracted from work of Liu et al. ${ }^{3}$

\begin{tabular}{|c|c|c|c|c|}
\hline \multirow{4}{*}{ Configuration } & \multirow{2}{*}{ Source } & \multicolumn{3}{|c|}{ Frequency $\left(\mathrm{cm}^{-1}\right)$} \\
\cline { 3 - 5 } & & $\mathrm{A}_{\mathrm{g}}{ }^{1}$ & $\mathrm{~B}_{2 \mathrm{~g}}$ & $\mathrm{~A}_{\mathrm{g}}{ }^{2}$ \\
\hline \multirow{4}{*}{ Bulk } & Expt $^{48}$ & 361.9 & 438.7 & 466.1 \\
\cline { 2 - 5 } & Expt & 361.2 & 438.2 & 465.7 \\
\cline { 2 - 5 } & $\mathrm{PBE}+\mathrm{D} 2$ & 357 & 425 & 454 \\
\cline { 2 - 5 } & $\mathrm{PBE}+\mathrm{D} 3$ & 351 & 430 & 450 \\
\hline \multirow{4}{*}{ Monolayer } & $\mathrm{Expt}{ }^{3}$ & 360.8 & 438.7 & 469.1 \\
\cline { 2 - 5 } & $\mathrm{PBE}{ }^{39}$ & 343.0 & 426.0 & 451.0 \\
\cline { 2 - 5 } & $\mathrm{PBE}+\mathrm{D} 2$ & 354 & 423 & 456 \\
\cline { 2 - 5 } & $\mathrm{PBE}+\mathrm{D} 3$ & 347 & 426 & 451 \\
\hline
\end{tabular}

Thus analysis of Raman active modes of black phosphorus and phosphorene suggests that the Grimme D2 correction provides results slightly closer to experiment with respect to the D3 version. For this reason, the Raman features of subsequent phosphorus species are treated with PBE+D2 theory level. In both cases the peak shift trends from bulk to monolayer show good reproduction of experimental shifts.

\subsubsection{Hittorf's Phosphorus (bulk and monolayer)}


Hittorf's phosphorus (sometimes also known as violet or red-V phosphorus) is a semiconducting layered allotrope ${ }^{51,52}$, structurally related to fibrous red phosphorus. Using Baudler's notation ${ }^{53}$, the atomic structure of Hittorf's phosphorus is composed of ]P2[P8]P2[P9] nanorods with pentagonal cross-section. The [P9] groups have a dangling bond which allows cross-linking between nanorods. In fibrous red-P these rods are parallel and cross-link in pairs, whereas in Hittorf's phosphorus the nanorods are arranged in bilayers, and in each layer the rods are perpendicular to the layer below. This allows the two layers to covalently cross-link into a bilayer grid of nanorods, with these bilayers then stacked on each other with weak van der Waals interaction. Recent experiment based on X-ray diffraction combined with different imaging analysis tools (such as high resolution transmission electron, scanning electron and atomic force microscopies) has refined the crystal structure compared to earlier literature, showing that the lattice is monoclinic with a space group of $P 2 / c^{52}$. Our DFT-D2 lattice vectors of $a=9.232 \AA, b=9.142 \AA, c=21.746 \AA$, and $\beta=$ $97.959^{\circ}$ are in excellent agreement with the literature experiment ${ }^{51,52}$ (see Table 4).

Table 4. PBE + D2 optimized unit cell lattice vectors of Hittorf's phosphorus compared with previous experiment ${ }^{51,52}$.

\begin{tabular}{|c|c|c|c|c|}
\hline Ref. & $a(\AA)$ & $b(\AA)$ & $c(\AA)$ & $\beta\left(^{\circ}\right)$ \\
\hline Expt. $^{51}$ & 9.210 & 9.150 & 22.600 & 106.100 \\
\hline Expt. $^{52}$ & 9.210 & 9.128 & 21.893 & 97.776 \\
\hline Present Work & 9.232 & 9.142 & 21.746 & 97.959 \\
\hline
\end{tabular}

The significantly more complex crystal structure as compared to white and black phosphorus allotropes results unsurprisingly in a much more complex Raman spectrum. Recent experiment breaks down the Raman spectrum of Hittorf's P into three distinct regions on the basis of peak intensities ${ }^{52}$, as shown in Fig. 5 (black curve). The first region between 100-300 $\mathrm{cm}^{-1}$ exhibits signals of medium intensity. A second section from $300-400 \mathrm{~cm}^{-1}$ does not show any band. The third last zone is characterized by intense peaks ranging from $358-471 \mathrm{~cm}^{-1}$. All of these peaks are computationally reproduced using our approach (Fig. 5, red curve, Table 4), although as for black phosphorus, their frequencies are slightly underestimated due to the DFT accuracy level. 

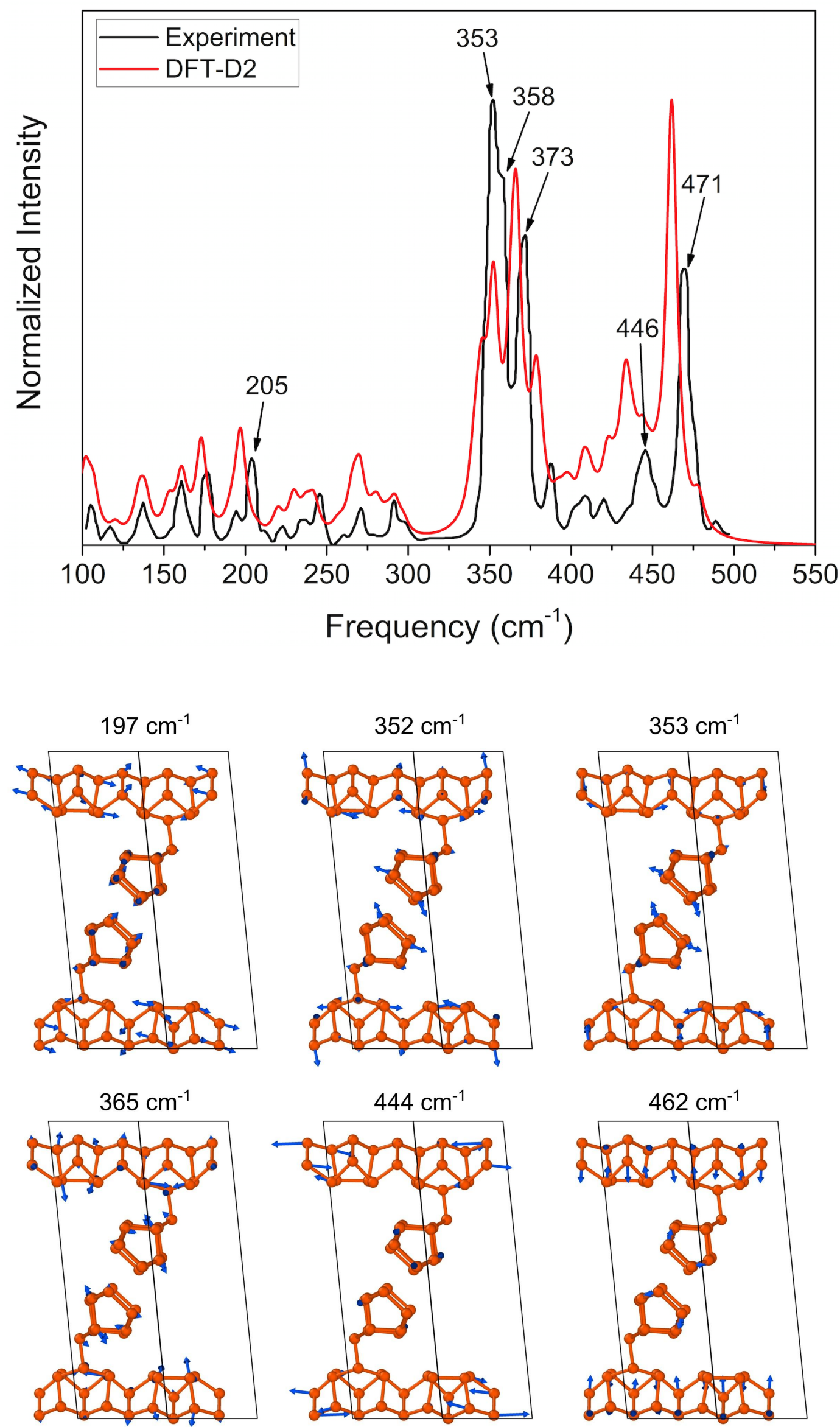
Figure 5: (Top) Raman spectrum of bulk red Hittorf's phosphorus (red) current DFT-D2 calculations at $2.3 \mathrm{eV}$ excitation energy $(538 \mathrm{~nm})$, (black) experimental data recorded by Zhang et al. ${ }^{52}$, primary values indicated by arrows. (Bottom) Calculated eigenvectors of key Raman active modes. Blue arrows indicate amplitude and direction of the displacements. Associated frequency is given in wavenumbers. Black lines indicate the symmetry of the simulated unit cell.

Table 4. Primary Raman active frequencies $\left(\mathrm{cm}^{-1}\right)$ in bulk red Hittorf's phosphorus of experimental measurements ${ }^{52}$ and first-principle $\mathrm{PBE}+\mathrm{D} 2$ calculations under $2.3 \mathrm{eV}$ excitation energy.

\begin{tabular}{|c|c|}
\hline \multicolumn{2}{|c|}{ Frequency $\left(\mathrm{cm}^{-1}\right)$} \\
\hline Expt. $\left(\right.$ Zhang $\left.^{52}\right)$ & PBE+D2 \\
\hline 205 & 197 \\
\hline 353 & 352 \\
\hline 358 & 366 \\
\hline 373 & 379 \\
\hline 446 & 444 \\
\hline 471 & 462 \\
\hline
\end{tabular}

Since Hittorf's phosphorus consists of stacked bilayers, it has also been proposed that these layers could be isolated ${ }^{46,52}$. These layers (named "hittorfene" amongst others) are composed of a 2D bilayer network of covalently cross-linked tubular fibers. The unit cell is orthorhombic with lattice vectors: $a=9.138(9.146) \AA, \mathrm{b}=9.201(9.203) \AA$, and $\gamma=90^{\circ}$ using $\mathrm{PBE}+\mathrm{D} 2(3)$ theory level. The electronic structure shows a direct band gap with transition at the Z point (see Supplementary Information, Section 2, Fig. S2). Interestingly, the calculated energy gap found for single-layer Hittorf's $\mathrm{P}(1.70 \mathrm{eV})$ is much wider compared to its black counterpart $(0.89 \mathrm{eV})$. These resulting values are quite close to those reported in literature ${ }^{46,54}$, as described in Table S2 reported in Section 1 of Supplementary Information. The direct character of the band gap has been double-checked using the Grimme D3 version and we found no changes. 
As far as we are aware no Raman characterization of Hittorfene has been reported to date. Fig. S7 shows our calculated PBE+D2 Raman spectrum of 2D Hittorfene P compared with the $3 \mathrm{D}$ bulk counterpart. The two spectra are broadly similar with only minor changes in peak intensities and positions. Thus we conclude that it will be difficult to utilise Raman to distinguish whether monolayer Hittorfene has been isolated.

\subsubsection{Blue Phosphorus (bulk and monolayer)}

Pressure-dependent Raman characterization shows that black phosphorus is subject to a structural transition into the blue phase at $\sim 4.2 \mathrm{GPa}^{44}$. Both phases consist of layers of buckled hexagonal monolayers, with the difference in the local ordering of the buckling (black phosphorus out of plane displacements form parallel lines, in blue phosphorus the atoms displace out of plane in alternating directions). The thermodynamic stability of blue phosphorus and its monolayer version blue phosphorene have been explored theoretically on different noble metal surfaces ${ }^{55,56}$, with interest in its electronic properties. Our PBE+D2 analysis shows semiconducting behavior for monolayer blue phosphorene with an indirect energy gap of $1.91 \mathrm{eV}$ (Supplementary Information, Section 2, see Fig. S3), close to literature values $^{11}$.

Raman measurements of blue phosphorene have not been reported to date, presumably because this phase is challenging to synthesize and observe experimentally. Our calculated Raman spectrum (Fig. 6) shows that monolayer blue phosphorene is characterized by only two intense Raman peaks, reflecting the higher symmetry of blue phosphorene as compared to its black counterpart. The first $\mathrm{E}_{\mathrm{g}}$ mode at $422 \mathrm{~cm}^{-1}$ corresponds to longitudinal displacements over in plane-directions, while the second $A_{1 \mathrm{~g}}$ mode at $534 \mathrm{~cm}^{-1}$ comes from out-of-plane vibrations, as shown in the inset. We note that previously literature calculated values are slightly higher (435 and $\left.550 \mathrm{~cm}^{-1}\right)^{39}$, possibly due to different $k$-point grid densities.

This Raman response is quite distinct from monolayer black phosphorene and suggests Raman is a suitable tool for distinguishing experimentally between the two. While the stronger $422 \mathrm{~cm}^{-1}$ peak is quite close to one of the calculated black phosphorene peak positions, the $534 \mathrm{~cm}^{-1}$ mode falls in a frequency range where no Raman signal is observed in any of the standard phosphorus phases considered above, and as such should be a 
characteristic blue phosphorus signature. Due to its out-of-plane nature, we expect this higher frequency peak to be the most sensitive to substrate interaction ${ }^{55,56}$. It will also be the mode most influenced by interlayer interaction when stacking blue phospherene, and as such its frequency might serve as a useful indicator for the number of layers, similarly to the $\mathrm{A}_{\mathrm{g}}{ }^{2}$ mode in black phosphorus ${ }^{3,50}$.

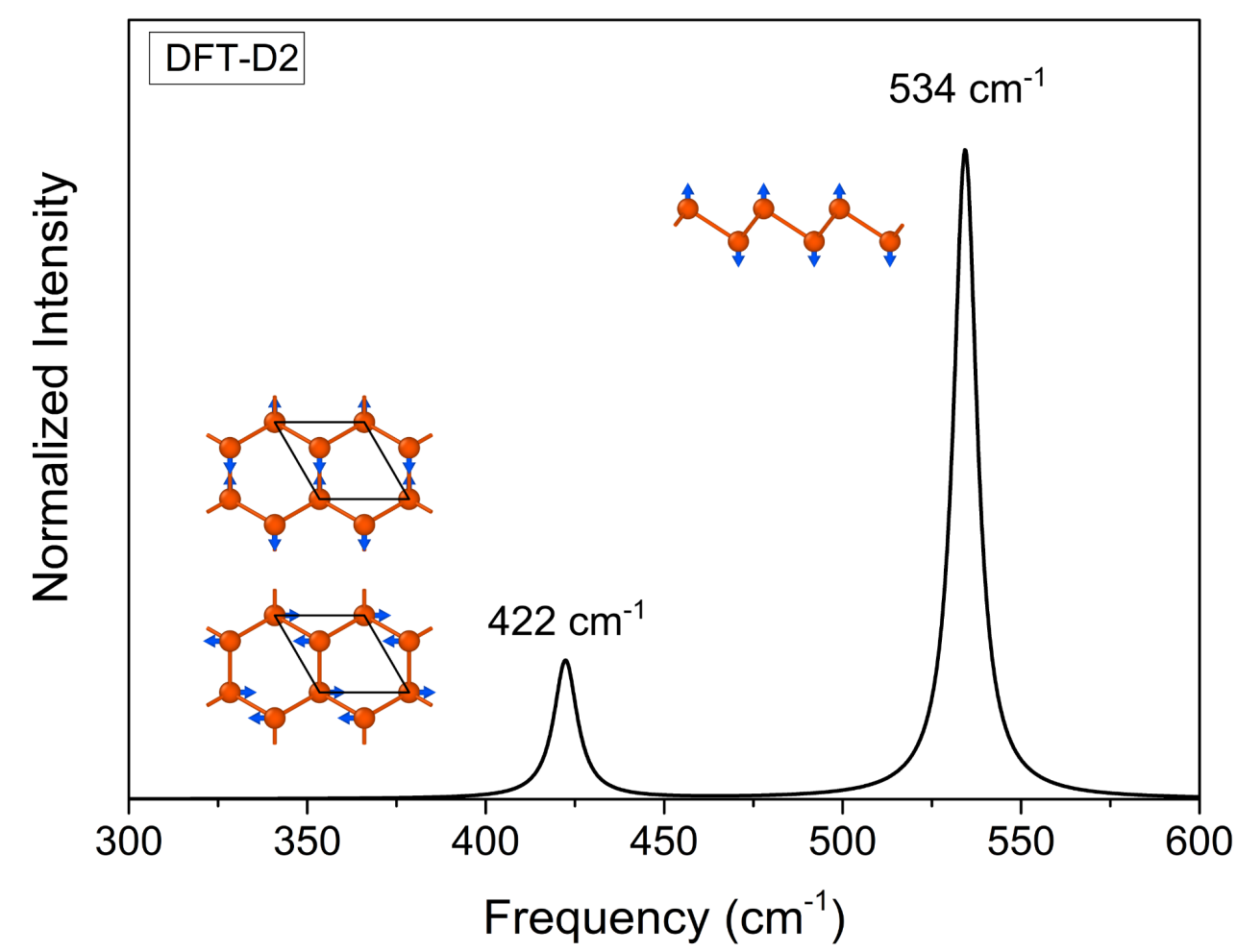

Figure 6: DFT-D2 calculated (red line) Raman spectrum of blue phosphorene at $2.3 \mathrm{eV}$ excitation energy $(538 \mathrm{~nm})$. Calculated eigenvectors of key Raman active modes shown in the inset. Blue arrows indicate amplitude and direction of the displacements. Associated frequency is given in wavenumbers. Black lines indicate the symmetry of the simulated unit cell.

\subsubsection{D-Phosphorus chain phases}

Since we have demonstrated above the reliability of our approach for accurately simulating Raman spectra of phosphorus allotropes, we next apply it to 1D phosphorus chain structures for which there is currently no experimental data available. Their existence remains somewhat controversial, and Raman spectroscopy may prove a useful characterisation tool. Recent experiments based on the encapsulation of white tetra-phosphorus inside carbon 
nanotube sheaths suggests the formation of such novel 1D-phases, which cannot exist when free-standing ${ }^{17}$. TEM imaging analysis suggests the formation of zig-zag phosphorus ladder chains when the nanotube diameter approaches $1 \mathrm{~nm}^{17}$. Other related phases may also be possible with different nanotube diameters. Amorphous phosphorus is also believed to consist of linear chain structures, and a range of structures have been proposed since the 1950s including zigzag chains ${ }^{17,53,57}$ and fused tetrahedra ${ }^{58}$. These chains have been isolated on surfaces ${ }^{59}$ and Raman could prove a very useful discriminatory tool in this case.

We first analyse the energetic stability of five chain configurations investigated by Hart et $a l^{17}$. Carbon nanotubes are not included in this set of calculations, i.e. phosphorus structures are free-standing. Our DFT-D analysis predicts the "trans" and "cis-trans" butterfly structures as the most stable configurations. Raman spectra for these structures are shown in Fig. 7, eigenvectors of the most intense Raman bands are given in Fig. 8. We note that confinement within extremely small diameter SWNTs may shift these values if chains are straightened and bond angles and lengths change as a result.

For the single zig-zag chain (Figure 8a) the strongest peak at $296 \mathrm{~cm}^{-1}$ corresponds to P-P stretch along the longitudinal direction. The alternating ladder configuration is characterized by three peaks (Figure $8 \mathrm{~b}$ ). The first and most intense band at $141 \mathrm{~cm}^{-1}$ shows compression and elongation modes along the chain direction. Since both structures only show a single strong Raman peak at quite low frequencies, this may be a tool to distinguish them experimentally.

In the zig-zag ladder structure (Figure 8c), the peaks at 151 and $427 \mathrm{~cm}^{-1}$ correspond to out-of-plane stretching of alternate P2 while those at 267, 408, and $457 \mathrm{~cm}^{-1}$ correspond to P pair displacements parallel and orthogonal to the chain axis. Since the primary peaks lie in a similar range to black phosphorus (since the eigenvectors show similar wagging motion, see Figure 9) this phase will likely be hard to distinguish by Raman.

The eigenmodes of the Raman active mode at $578 \mathrm{~cm}^{-1}$ for the trans-butterfly chain, as well as the peaks at 548 and $561 \mathrm{~cm}^{-1}$ of the cis-trans butterfly structure, are similar to those observed for $\mathrm{A}_{1}$ peak of bulk white $\mathrm{P}\left(594 \mathrm{~cm}^{-1}\right)$. This breathing mode is characteristic of a P4 tetrahedral cage, and its downshift in the chains is a signature of polymerisation and associated bonding between the tetrahedra. 
CNT confinement has also resulted in microscopy images of phosphorus with apparently square columnar structure ${ }^{19}$. The twisted square columnar phosphorus structure shows semiconducting behaviour with an indirect band gap $1.88 \mathrm{eV}$ (Supplementary Information, Fig. S4), comparable with that of blue phosphorene (1.91eV). Its calculated Raman spectrum has a primary low frequency peak at $147 \mathrm{~cm}^{-1}$ (Fig. S8) and is quite similar to the alternating ladder case, consistent with their structural similarity. Weaker peaks at 359 and $396 \mathrm{~cm}^{-1}$ are associated with the square motifs.

While various amorphous structures may exist depending on sample preparation, literature Raman spectra for amorphous thin films ${ }^{60}$ show little resemblance to any of these calculated spectra, matching more closely that of Hittorf's phosphorus. We suggest therefore that the linear chains randomly oriented in amorphous phosphorus are more likely to be P8]P2 and P9]P2 structures seen in Hittorf's and fibrous red phosphorus rather than the chains considered here. This would also be consistent with the enhanced energetic stability of the P8]P2/P9]P2 chains. 


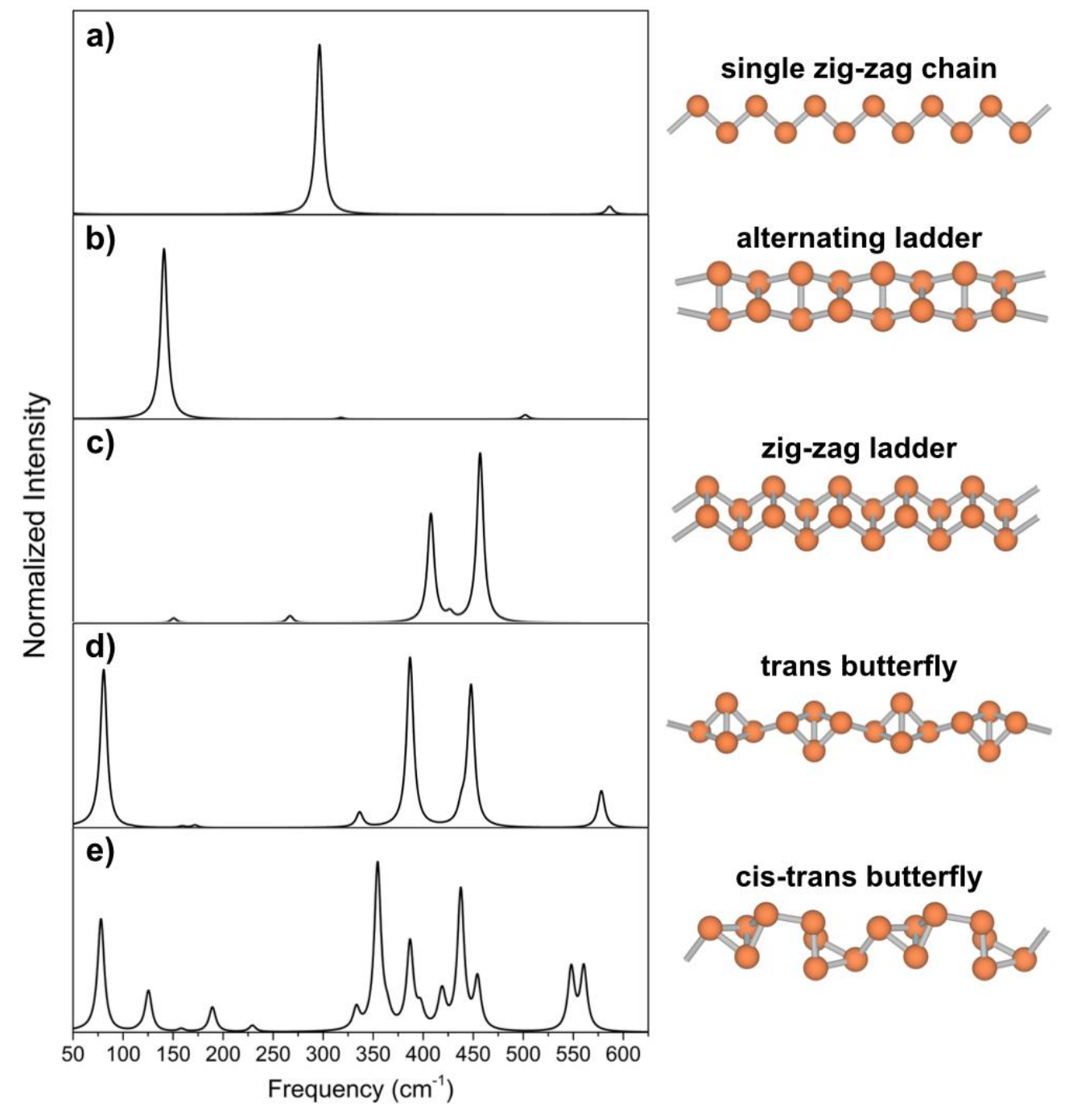

Figure 7: (Left) DFT-D2 calculated (red line) Raman spectrum of one-dimensional phosphorus structures at $2.3 \mathrm{eV}$ excitation energy (538 nm). (Right) Geometry optimised chain structures: (a) single zig-zag chains, (b) alternating ladder, (c) zig-zag ladder, (d) trans butterfly, (e) cis-trans butterfly. 


a)

Figure 8: Representation of DFT-D2 calculated phonon eigenvectors of the most intense Raman peaks for the: (a) single zig-zag chains, (b) alternating ladder, (c) zig-zag ladder, (d) trans butterfly, (e) cis-trans butterfly at $2.3 \mathrm{eV}$ excitation energy (538 $\mathrm{nm})$. Associated frequency is given in wavenumbers and can be compared with Fig. 7.

\section{Conclusions}

We have systematically studied the energetic stability and the Raman spectra of bulk and layered phosphorus allotropes, as well as various theoretically proposed 1D-polymerised structures. Hittorf's phosphorus is the most stable form using Grimme DFT-D3 approximation, confirming prior literature via Tkatchenko-Sheffler and RPA approaches.

Using an implementation of the first-order semi-classical Placzek approximation, Raman spectra were simulated including all possible directions of the incident light and all polarization vectors of input/output photons at a $2.3 \mathrm{eV}$ excitation wavelength. The 
methodology can be readily extended to other excitation energies. Although DFT-D3 provided more reliable results for the relative stability of bulk phosphorus allotropes, the simulated peak positions show better agreement with experiment when calculated at the DFT-D2 theory level. This is particularly evident for black phosphorus.

This approach for determining Raman intensities is relatively simplistic. Its speed and scalability allows handling of large systems not tractable with more complete methods. Despite its simplicity it accurately reproduces spectra for a wide range of phosphorus phases and successfully detects quite subtle experimentally observed spectral features, such as crystal symmetry induced peak splitting in low frequency peaks of white phosphorus, and the dispersive peak behaviour of black $\mathrm{P}$ going from 3D bulk to 2D monolayer phase.

Having demonstrated the reliability of this approach with experimentally observed bulk phases, we have extended the method to a range of monolayer phosphorus species (black, red and blue), and finally to a selection of 1D-chain structures proposed theoretically but not experimentally confirmed to date. Notably this data can be compared with experimental Raman spectra for bulk amorphous phosphorus. This comparison suggests that most of these small chain models can be eliminated as structural units for the amorphous phase, which shows much better match to structural units from red-P.

\section{Associated content}

\section{Supporting Information}

The Supporting Information is available in the online version of the paper.

Energy stability, geometry and electronic parameters of Phosphorus allotropes. Electronic structures of Phosphorus allotropes. Raman spectra of Phosphorus allotropes.

Atomic structure of blue phosphorene.

Atomic structures of single zig-zag chain, alternating ladder, zig-zag ladder, trans butterfly, cis-trans butterfly, and twisted square chains.

\section{Author Information}




\title{
Corresponding Authors
}

\author{
*E-mail: chris.ewels@cnrs-imn.fr \\ *E-mail: rybkovskiyd@gmail.com
}

\section{Notes}

The authors declare no conflict of interest.

\section{Acknowledgements}

The work leading to these results received funding from the French Agence Nationale de Recherche Projects "EdgeFiller" and "OPIFCat". We thank Lyuba Bulusheva for fruitful discussion and useful comments. A. Impellizzeri, C. P. Ewels and D. V. Rybkovskiy acknowledge the CCIPL "Centre de Calcul Intensif Pays de la Loire" where many of the calculations were performed. D. V. Rybkovskiy acknowledges support from Russian Ministry of Science and Higher Education (Grant No. 2711.2020.2 to leading scientific schools).

\section{References}

(1) Schlesinger, M. E. The Thermodynamic Properties of Phosphorus and Solid Binary Phosphides. Chem. Rev. 2002, 102 (11), 4267-4302. https://doi.org/10.1021/cr000039m.

(2) Xu, Y.; Shi, Z.; Shi, X.; Zhang, K.; Zhang, H. Recent Progress in Black Phosphorus and Black-Phosphorus-Analogue Materials: Properties, Synthesis and Applications. Nanoscale 2019, 11 (31), 14491-14527. https://doi.org/10.1039/C9NR04348A.

(3) Liu, H.; Neal, A. T.; Zhu, Z.; Luo, Z.; Xu, X.; Tománek, D.; Ye, P. D. Phosphorene: An Unexplored 2D Semiconductor with a High Hole Mobility. ACS Nano 2014, 8 (4), 4033-4041. https://doi.org/10.1021/nn501226z.

(4) O'hare, P. A. G.; Lewis, B. M.; Shirotani, I. Thermodynamic Stability of Orthorhombic Black Phosphorus. Thermochim. Acta 1988, 129 (1), 57-62.

https://doi.org/10.1016/0040-6031(88)87196-X.

(5) Brazhkin, V. V.; Zerr, A. Ju. Relative Stability of Red and Black Phosphorus at $\mathrm{P}<1$ GPa. J. Mater. Sci. 1992, 27 (10), 2677-2681. https://doi.org/10.1007/BF00540689. 
(6) Simon, A.; Borrmann, H.; Horakh, J. On the Polymorphism of White Phosphorus. Chem. Ber. 1997, 130 (9), 1235-1240. https://doi.org/10.1002/cber.19971300911.

(7) Ruck, M.; Hoppe, D.; Wahl, B.; Simon, P.; Wang, Y.; Seifert, G. Fibrous Red Phosphorus. Angew. Chem. Int. Ed. 2005, 44 (46), 7616-7619. https://doi.org/10.1002/anie.200503017.

(8) Jamieson, J. C. Crystal Structures Adopted by Black Phosphorus at High Pressures. Science 1963, 139 (3561), 1291-1292. https://doi.org/10.1126/science.139.3561.1291.

(9) Zhu, Z.; Tománek, D. Semiconducting Layered Blue Phosphorus: A Computational Study. Phys Rev Lett 2014, 112 (17), 176802.

https://doi.org/10.1103/PhysRevLett.112.176802.

(10) Zhang, J. L.; Zhao, S.; Han, C.; Wang, Z.; Zhong, S.; Sun, S.; Guo, R.; Zhou, X.; Gu, C. D.; Yuan, K. D.; Li, Z.; Chen, W. Epitaxial Growth of Single Layer Blue Phosphorus: A New Phase of Two-Dimensional Phosphorus. Nano Lett. 2016, 16 (8), 4903-4908.

https://doi.org/10.1021/acs.nanolett.6b01459.

(11) Karttunen, A. J.; Linnolahti, M.; Pakkanen, T. A. Icosahedral and Ring-Shaped Allotropes of Phosphorus. Chem. - Eur. J. 2007, 13 (18), 5232-5237.

https://doi.org/10.1002/chem.200601572.

(12) Wu, M.; Fu, H.; Zhou, L.; Yao, K.; Zeng, X. C. Nine New Phosphorene Polymorphs with Non-Honeycomb Structures: A Much Extended Family. Nano Lett. 2015, 15 (5), 3557-3562. https://doi.org/10.1021/acs.nanolett.5b01041.

(13) Zhuo, Z.; Wu, X.; Yang, J. Two-Dimensional Phosphorus Porous Polymorphs with Tunable Band Gaps. J. Am. Chem. Soc. 2016, 138 (22), 7091-7098.

https://doi.org/10.1021/jacs.6b02964.

(14) Deringer, V. L.; Pickard, C. J.; Proserpio, D. M. Hierarchically Structured Allotropes of Phosphorus from Data-Driven Exploration. Angew. Chem. Int. Ed. 2020, 59 (37), 15880-15885. https://doi.org/10.1002/anie.202005031.

(15) Deringer, V. L.; Caro, M. A.; Csányi, G. A General-Purpose Machine-Learning Force Field for Bulk and Nanostructured Phosphorus. Nat. Commun. 2020, 11 (1), 5461.

https://doi.org/10.1038/s41467-020-19168-Z.

(16) Guo, H.; Lu, N.; Dai, J.; Wu, X.; Zeng, X. C. Phosphorene Nanoribbons, Phosphorus Nanotubes, and van Der Waals Multilayers. J. Phys. Chem. C 2014, 118 (25), 14051-14059. https://doi.org/10.1021/jp505257g.

(17) Hart, M.; White, E. R.; Chen J.; McGilvery, C. M.; Pickard; Michaelides, A.; Sella, A.; Shaffer, M. S. P.; Salzmann, C. G. Encapsulation and Polymerization of White Phosphorus Inside Single-Wall Carbon Nanotubes. Angew. Chem. Int. Ed. 2017, 56 (28), 8144-8148. https://doi.org/10.1002/anie.201703585. 
(18) Hart, M.; Chen, J.; Michaelides, A.; Sella, A.; Shaffer, M. S. P.; Salzmann, C. G. One-dimensional Pnictogen Allotropes inside Single-Wall Carbon Nanotubes. Z. Inorg. Chem. 2019, 58 (22), 15216-15224. https://doi.org/10.1021/acs.inorgchem.9b02190.

(19) Zhang, J.; Fu, C.; Song, S.; Du, H.; Zhao, D.; Huang, H.; Zhang, L.; Guan, J.; Zhang, Y.; Zhao, X.; Ma, C.; Jia, C.-L.; Tománek, D. Changing the Phosphorus Allotrope from a Square Columnar Structure to a Planar Zigzag Nanoribbon by Increasing the Diameter of Carbon Nanotube Nanoreactors. Nano Lett. 2020, 20 (2), 1280-1285.

https://doi.org/10.1021/acs.nanolett.9b04741.

(20) Aykol, M.; Doak, J. W.; Wolverton, C. Phosphorus Allotropes: Stability of Black versus Red Phosphorus Re-Examined by Means of the van Der Waals Inclusive Density Functional Method. Phys Rev B 2017, 95 (21), 214115.

https://doi.org/10.1103/PhysRevB.95.214115.

(21) Stephenson, C. C.; Potter, R. L.; Maple, T. G.; Morrow, J. C. The Thermodynamic Properties of Elementary Phosphorus The Heat Capacities of Two Crystalline Modifications of Red Phosphorus, of $\alpha$ and $\beta$ White Phosphorus, and of Black Phosphorus from 15 to 300 K. J. Chem. Thermodyn. 1969, 1 (1), 59-76. https://doi.org/10.1016/0021-9614(69)90037-8.

(22) Long, D. A. The Raman Effect: A Unified Treatment of the Theory of Raman Scattering by Molecules. 2002.

(23) Winterauer, D. J.; Funes-Hernando, D.; Duvail, J.-L.; Moussaoui, S.; Batten, T.; Humbert, B. Sub-Micron Spatial Resolution in Far-Field Raman Imaging Using Positivity-Constrained Super-Resolution. Appl Spectrosc 2019, 73 (8), 902-909.

(24) Winterauer, D. J.; Funes-Hernando, D.; Duvail, J.-L.; Moussaoui, S.; Batten, T.; Humbert, B. Nanoscale Spatial Resolution in Far-Field Raman Imaging Using Hyperspectral Unmixing in Combination with Positivity Constrained Super-Resolution. Appl. Spectrosc. 2020, 74 (7), 780-790. https://doi.org/10.1177/0003702820920688.

(25) Torche, A.; Mauri, F.; Charlier, J.-C.; Calandra, M. First-Principles Determination of the Raman Fingerprint of Rhombohedral Graphite. Phys Rev Mater. 2017, 1 (4), 041001. https://doi.org/10.1103/PhysRevMaterials.1.041001.

(26) Taghizadeh, A.; Leffers, U.; Pedersen, T. G.; Thygesen, K. S. A Library of Ab Initio Raman Spectra for Automated Identification of 2D Materials. Nat. Commun. 2020, 11 (1), 3011. https://doi.org/10.1038/s41467-020-16529-6.

(27) Picheau, E.; Impellizzeri, A.; Rybkovskiy, D.; Bayle, M.; Mevellec, J.-Y.; Hof, F.; Saadaoui, H.; Noé, L.; Torres Dias, A. C.; Duvail, J.-L.; Monthioux, M.; Humbert, B.; Puech, P.; Ewels, C. P.; Pénicaud, A. Intense Raman D Band without Disorder in Flattened Carbon Nanotubes. ACS Nano 2021, 15 (1), 596-603. https://doi.org/10.1021/acsnano.0c06048. 
(28) Walter, M.; Moseler, M. Ab Initio Wavelength-Dependent Raman Spectra: Placzek Approximation and Beyond. J. Chem. Theory Comput. 2020, 16 (1), 576-586. https://doi.org/10.1021/acs.jctc.9b00584.

(29) Rayson, M. J.; Briddon, P. R. Rapid Iterative Method for Electronic-Structure Eigenproblems Using Localised Basis Functions. Comput. Phys. Commun. 2008, 178 (2), 128-134. https://doi.org/10.1016/j.cpc.2007.08.007.

(30) Rayson, M. J.; Briddon, P. R. Highly Efficient Method for Kohn-Sham Density Functional Calculations of 500-10 000 Atom Systems. Phys. Rev. B 2009, 80 (20), 205104. https://doi.org/10.1103/PhysRevB.80.205104.

(31) Rayson, M. J. Rapid Filtration Algorithm to Construct a Minimal Basis on the Fly from a Primitive Gaussian Basis. Comput. Phys. Commun. 2010, 181 (6), 1051-1056. https://doi.org/10.1016/i.cpc.2010.02.012.

(32) Perdew, J. P.; Burke, K.; Ernzerhof, M. Generalized Gradient Approximation Made Simple. Phys. Rev. Lett. 1996, 77 (18), 3865-3868. https://doi.org/10.1103/PhysRevLett.77.3865.

(33) Grimme, S. Semiempirical GGA-Type Density Functional Constructed with a Long-Range Dispersion Correction. J. Comput. Chem. 2006, 27 (15), 1787-1799. https://doi.org/10.1002/jcc.20495.

(34) Grimme, S.; Antony, J.; Ehrlich, S.; Krieg, H. A Consistent and Accurate Ab Initio Parametrization of Density Functional Dispersion Correction (DFT-D) for the 94 Elements H-Pu. J. Chem. Phys. 2010, 132 (15), 154104. https://doi.org/10.1063/1.3382344.

(35) Hartwigsen, C.; Goedecker, S.; Hutter, J. Relativistic Separable Dual-Space Gaussian Pseudopotentials from H to Rn. Phys. Rev. B 1998, 58 (7), 3641-3662.

https://doi.org/10.1103/PhysRevB.58.3641.

(36) Shaw, J. G. M.; Briddon, P. R. Marker-Method Calculations for Electrical Levels Using Gaussian-Orbital Basis Sets. In Theory of defects in semiconductors; Springer, 2007; pp 69-94.

(37) Stukowski, A. Visualization and analysis of atomistic simulation data with OVITO-the Open Visualization Tool. Modelling. Simul. Mater. Sci. Eng. 2010, 18 (1), 015012. https://doi.org/10.1088/0965-0393/18/1/015012.

(38) Borin Barin, G.; Fairbrother, A.; Rotach, L.; Bayle, M.; Paillet, M.; Liang, L.; Meunier, V.; Hauert, R.; Dumslaff, T.; Narita, A.; Müllen, K.; Sahabudeen, H.; Berger, R.; Feng, X.; Fasel, R.; Ruffieux, P. Surface-Synthesized Graphene Nanoribbons for Room Temperature Switching Devices: Substrate Transfer and Ex Situ Characterization. ACS Appl. Nano Mater. 2019, 2 (4), 2184-2192. https://doi.org/10.1021/acsanm.9b00151. 
(39) Deng, Z.; Li, Z.; Wang, W.; She, J. Vibrational Properties and Raman Spectra of Pristine and Fluorinated Blue Phosphorene. Phys Chem Chem Phys 2019, 21 (3), 1059-1066. https://doi.org/10.1039/C8CP05699D.

(40) Okudera, H.; Dinnebier, R. E.; Simon, A. The crystal structure of $\gamma$-P4, a low temperature modification of white phosphorus. Z. Kristallogr. 2005, 220 (2-3), 259-264. https://doi.org/10.1524/zkri.220.2.259.59137.

(41) Östmark, H.; Wallin, S.; Hore, N.; Launila, O. Raman spectra of $P_{4}$ at low temperatures. J. Chem. Phys. 2003, 119 (12), 5918-5922. https://doi.org/10.1063/1.1602062.

(42) Rissi, E. N.; Soignard, E.; McKiernan, K. A.; Benmore, C. J.; Yarger, J. L. Pressure-Induced Crystallization of Amorphous Red Phosphorus. Solid State Commun. 2012, 152 (5), 390-394. https://doi.org/10.1016/j.ssc.2011.12.003.

(43) Keyes, R. W. The Electrical Properties of Black Phosphorus. Phys Rev 1953, 92 (3), 580-584. https://doi.org/10.1103/PhysRev.92.580.

(44) Warschauer, D. Electrical and Optical Properties of Crystalline Black Phosphorus. J. Appl. Phys. 1963, 34 (7), 1853-1860. https://doi.org/10.1063/1.1729699.

(45) Ribeiro, H. B.; Pimenta, M. A.; de Matos, C. J. S. Raman Spectroscopy in Black Phosphorus. J. Raman Spectrosc. 2018, 49 (1), 76-90. https://doi.org/10.1002/jrs.5238.

(46) Schusteritsch, G.; Uhrin, M.; Pickard, C. J. Single-Layered Hittorf's Phosphorus: A Wide-Bandgap High Mobility 2D Material. Nano Lett. 2016, 16 (5), 2975-2980. https://doi.org/10.1021/acs.nanolett.5b05068.

(47) Cai, Y.; Zhang, G.; Zhang, Y.-W. Layer-Dependent Band Alignment and Work Function of Few-Layer Phosphorene. Sci. Rep. 2014, 4 (1), 6677. https://doi.org/10.1038/srep06677.

(48) Kundu, A.; Tristant, D.; Sheremetyeva, N.; Yoshimura, A.; Torres Dias, A.; Hazra, K. S.; Meunier, V.; Puech, P. Reversible Pressure-Induced Partial Phase Transition in Few-Layer Black Phosphorus. Nano Lett. 2020, 20 (8), 5929-5935.

https://doi.org/10.1021/acs.nanolett.0c01784.

(49) Suryawanshi, S. R.; More, M. A.; Late, D. J. Laser Exfoliation of 2D Black Phosphorus Nanosheets and Their Application as a Field Emitter. RSC Adv 2016, 6 (113), 112103-112108. https://doi.org/10.1039/C6RA24526A.

(50) Guo, Z.; Zhang, H.; Lu, S.; Wang, Z.; Tang, S.; Shao, J.; Sun, Z.; Xie, H.; Wang, H.; Yu, X.-F.; Chu, P. K. From Black Phosphorus to Phosphorene: Basic Solvent Exfoliation, Evolution of Raman Scattering, and Applications to Ultrafast Photonics. Adv. Funct. Mater. 2015, 25 (45), 6996-7002. https://doi.org/10.1002/adfm.201502902. 
(51) Thurn, H. and Krebs, H. Uber Struktur und Eigenschaften der Halbmetalle. XXII. Die Kristallstruktur des Hittorfschen Phosphors. Acta. Cryst. B. 1969, 25, 125-135. https://doi.org/10.1107/S0567740869001853.

(52) Zhang, L.; Huang, H.; Zhang, B.; Gu, M.; Zhao, D.; Zhao, X.; Li, L.; Zhou, J.; Wu, K.; Cheng, Y.; Zhang, J. Structure and Properties of Violet Phosphorus and Its Phosphorene Exfoliation. Angew. Chem. Int. Ed. 2020, 59 (3), 1074-1080.

https://doi.org/10.1002/anie.201912761.

(53) Böcker, S.; Häser, M. Covalent Structures of Phosphorus: A Comprehensive Theoretical Study. Z. Für Anorg. Allg. Chem. 1995, 621 (2), 258-286.

https://doi.org/10.1002/zaac.19956210215.

(54) Lu, Y.-L.; Dong, S.; Zhou, W.; Dai, S.; Zhou, B.; Zhao, H.; Wu, P. ve Hittorf's violet phosphorene as a promising candidate for optoelectronic and photocatalytic applications: first-principles characterization. Phys. Chem. Chem. Phys. 2018, 20 (17), 11967-11975. https://doi.org/10.1039/C8CP01364K.

(55) Zhao, S.; Zhang, J. L.; Chen, W.; Li., Z. Structure of Blue Phosphorus Grown on Au(111) Surface Revisited. J. Phys. Chem. C 2020, 124 (3), 2024-2029. https://doi.org/10.1021/acs.jpcc.9b10511.

(56) Zhao, S.; Li., Z. Blue Phosphorus Growth on Different Noble Metal Surfaces: From a 2D Alloy Network to an Extended Monolayer. J. Phys. Chem. C 2021, 125 (1), 675-679. https://doi.org/10.1021/acs.jpcc.0c10478.

(57) Haeser, M.; Schneider, U; Ahlrichs, R. Cluster of phosphorus: a theoretical investigation. J. Am. Chem. Soc. 1992, 114 (24), 9551-9559. https://doi.org/10.1021/ja00050a039.

(58) Pauling, L.; Simonetta, M. Bond Orbitals and Bond Energy in Elementary Phosphorus. J. Chem. Phys. 1952, 20 (1), 29-34. https://doi.org/10.1063/1.1700191.

(59) Zhang, S.; Qian, H.-j.; Liu, Z.; Ju, H; Lu, Z.-y.; Zhang, H.; Chi, L.; Cui, S. Towards Unveiling the Exact Molecular Structure of Amorphous Red Phosphorus by Single-Molecule Studies. Angew. Chem. Int. Ed. 2019, 58 (6), 1659-1663.

https://doi.org/10.1002/anie.201811152.

(60) Olego, D. J.; Baumann, J. A.; Schachter, R. The microscope structures of amorphous phosphorus. Solid. State. Commun. 1985, 53 (11), 905-908.

https://doi.org/10.1016/0038-1098(85)90457-0. 